\title{
TOLERANCE TO DESICCATION STRESS IN CHIRONOMUS RAMOSUS THROUGH PLASTICITY IN HOMEOSTATIC CONTROL
}

\author{
LEENA THORAT and BIMALENDU B. NATH
}

\author{
Department of Zoology, Savitribai Phule Pune University, Pune-411007, India \\ * Corresponding author: bbnath@gmail.com
}

\begin{abstract}
Desiccating environments pose physiological challenges to organisms, especially those experiencing them on recurring basis. Several desiccation-tolerant organisms are known to withstand loss of body water in response to seasonal dehydration patterns through adaptations that facilitate their sustenance in the dry state followed by recovery upon return of favourable hydrating conditions. In the present study, we have chosen Chironomus ramosus, an aquatic midge species as a laboratory model system towards the exploration of its desiccation tolerance ability. We confirm that upon desiccation exposure at low relative humidity, larvae of $C$. ramosus exhibited rapid water loss and could revive upon rehydration. Moreover, the revived individuals underwent successful metamorphosis; albeit delayed. This heterochrony in the developmental calendar was experimentally verified by the investigation of ecdysone levels which suggested an altered pattern of ecdysis in response to the stress. These data suggested the organism's plasticity in developmental homeostasis when confronted with dehydration stress. Furthermore, spectrofluorometric assays indicated the occurrence of thiol damage in the larvae. Investigation of Hsp70, the evolutionarily conserved stress responsive gene suggested that $\mathrm{Hsp70}$ was indeed up-regulated during the rehydration period, thus facilitating recovery of the larvae from dehydration. In summary, several lines of evidence involving cellular, physiological and developmental adaptations revealed the ability of $C$. ramosus larvae to minimize the diverse categories of damage caused by desiccation stress through plasticity in their homeostatic mechanism.
\end{abstract}

Keywords: Chironomus ramosus, desiccation, developmental heterochrony, ecdysone, heat shock proteins, thiol

\section{Introduction}

Environmental stressors include a range of seasonal as well as climate change-related parameters that are capable of influencing the survival and distribution of organisms. Desiccation resulting from temperature-humidity fluctuations forms one of the predominant stressful factors in nature. The effects of water deprivation in aquatic insects are far more intense in comparison to non-aquatic insect groups (Chown et al. 2011). To overcome the problems of water deficits, desiccation tolerant organisms are known to increase the concentrations of selective biomolecules like non-reducing carbohydrates, amino acids and stress proteins e.g. heat shock proteins (HSPs) (for details see Tunnacliffe and Lapinski 2003; Benoit et al. 2010). These stress protectants are known to eliminate the effects of cellular damage thus facilitating the organism to cope exposures to environmental stresses. Hsp70, in particular, has been widely established across most plant and animal groups as a molecular chaperone that prevents the aggregation and folding/unfolding of other proteins (Zhao and Jones 2012). Thus, in most cases, the presence or absence of Hsp70 can be an important determining factor of cell survival. Although the role of the Hsp70 family in association to general stresses is well known, few reports have documented its connection to desiccation stress. In this study, we aim to explore the desiccation-responsive presence of Hsp70 in C. ramosus which is an evolutionarily conserved protein that is implicated in different stress-response conditions.
In most insects, alterations in both internal and external conditions are known to affect their temporal patterns of development (Raushenbach et al. 2004; Vandersmissen et al. 2014). In the perspective of environmental stresses, insect hormones have appeared to participate in various events regulating recovery and developmental mechanisms, in addition to their prime role in embryonic development (Smith 2003).Variations in hormonal titres including ecdysteroids and juvenile hormone $(\mathrm{JH})$ have been primarily identified in coordinating the developmental changes in response to external conditions that induce diapause in insects (Koštál 2006). Several studies have reported such observations in diapausing insects (such as Culex pipens, Manduca sexta, Omphisa fuscidentalis and Antheraea yamamai); however there is a dearth of similar information in insect species subjected to desiccation stress. Such biomarkers that reflect the physiological changes of an organism's internal conditions can be promising tools towards the better understanding of the finer interactions between organisms and their environments (Menge et al. 2002).

Chironomid midges are an abundant insect group of freshwater ecosystems and serve as an important food source for organisms in both aquatic and terrestrial habitats (Ferrington 2008). Owing to their robust physiology, midges have been a popular model system for the elucidation of cellular responses against different stressors e.g. heat, radiation, UV, toxicants and heavy metals (Choi and Ha 2009; Thorat and Nath 2010; Cao et al. 2013; Morais et al. 2014). In the past few decades, the use of midges for 
aquatic biomonitoring programmes has been increasingly evident (Rosenberg 1992). Chironomid midges inhabit ecosystems as diverse as freshwater bodies, temporary rock pools, wetlands and also the terrestrial biomes of Antarctica. During field surveys, we came across a population of Chironomus ramosus that experiences transient desiccation bouts especially during summers. The remarkable ability of this larval population to revive after spells of desiccation conditions prompted us to explore the capability of desiccation tolerance in the larvae. In this study, we used larvae of $C$. ramosus, a tropical midge to assess its desiccation tolerance ability and also explored a few of the physiological underpinnings involved therein. We investigated the developmental profile of the larvae in response to desiccation and identified the presence of Hsp70 that alleviates the damage inflicted due to water loss.

\section{Materials and Methods}

\section{Organisms}

Isofemale lines of $C$. ramosus were maintained under controlled laboratory conditions at $25 \pm 1{ }^{\circ} \mathrm{C}$ and with photoperiod cycle of $14 \mathrm{~h}$ light : $10 \mathrm{~h}$ darkness. Larvae were supplemented with food medium as described in Nath and Godbole (1998). Third instar larvae were used for all further experiments.

\section{Stress Treatment}

Larvae were exposed to desiccation stress inside a desiccator at $23 \pm 1^{\circ} \mathrm{C}$ as described in Thorat et al. (2012). In brief, larvae were collected in groups of 30 and thoroughly rinsed in water to get rid of the adhering substratum particles. Thereafter, larvae were blotted on filter paper and held on sterilized glass Petri dishes for $1 \mathrm{~h}$ inside a desiccator at $<5 \%$ relative humidity $(\mathrm{RH})$. The dried larvae were then rehydrated in water $(20 \mu \mathrm{l} /$ individual $)$ and allowed to revive under ambient conditions. Petri dishes were gradually filled up with water and larvae were allowed to recover under ambient conditions at $75 \pm 10 \%$ RH. Untreated larvae were used as controls.

\section{Life History Analysis}

Revived larvae were kept under observation in ambient conditions in order to track their life history post rehydration. The larval, pupal and adult life span post rehydration was compared to that with the untreated controls.

\section{Hormone Titre Measurements by Liquid Chromatography- Mass Spectrometry (LC-MS)}

Revived larvae were compared to those with the untreated controls for the measurement of ecdysone hormone titres using larval hemolymph according to a published protocol with minor modifications (Oostra et al. 2011). Larval hemolymph was added to $300 \mu$ of methanol: isooctane solution $(1: 1 \mathrm{v} / \mathrm{v})$ in $1.5 \mathrm{ml}$ centrifuge tubes and vortexed for $20 \mathrm{~s}$. Tubes were allowed to stand at room temperature for $20 \mathrm{~min}$ and then centrifuged at $10,000 \mathrm{~g}$ at $5^{\circ} \mathrm{C}$ for $20 \mathrm{~min}$. Supernatants were transferred to fresh centrifuge tubes and heated on a water bath to evaporate until dry. The precipitate was reconstituted in 1:1 methanol-water and injected into the autosampler (Shimadzu SIL-10ADVP) connected to an Eldex Micro Pro High Performance Liquid Chromatography (HPLC) system. Separation was carried out on a $150 \times 2 \mathrm{~mm} \mathrm{C18}$ reversed-phase column (ReproSil-Pur ODS-3) protected by a guard column (C18 cartridge, Phenomenex, Aschaffenburg, Germany) at a flow rate of $200 \mu \mathrm{min}^{-1}$ and column temperature of $37^{\circ} \mathrm{C}$. Mobile phase consisted of a methanol:water gradient varying between 30\% and 100\% over $20 \mathrm{~min}$. MS analysis was performed using electrospray ionisation in the positive mode on a Shimadzu LCMS-2010A and post-run analysis was performed using the Shimadzu LCMS solution software (Version 3.0). Known concentrations of commercially available 20-hydroxyecdysone (Abcam, ab4216) were used as standards. All solvents used were HPLC grade.

\section{Spectrofluorimetric Analyses}

Control, desiccated and rehydrated larvae were incubated for $15 \mathrm{~min}$ in 5-IAF dye (5-iodoacetamidofluorescein; Excitation/emission maxima $=494 / 518 \mathrm{~nm}$ ) solution at a concentration of $10 \mu \mathrm{M}$. Larvae were then homogenized in Phosphate buffered saline (PBS; pH 7.4) and centrifuged for $15 \mathrm{~min}$ at $4{ }^{\circ} \mathrm{C}$ at $10,000 \mathrm{~g}$. The supernatant was used to record readings on a spectrofluorometer (Shimadzu RF-5301).

\section{RNA Isolation and RT-qPCR}

RNA from whole larvae $(n=10)$ was isolated using the peqGOLD Total RNA Kit according to the manufacturer's instructions. DNAse I digestion step was included as part of the protocol and RNA was eluted at the final step with $50 \mu \mathrm{l}$ of nuclease free water. Following quantification, $1 \mu \mathrm{g}$ of the total isolated RNA was used for cDNA synthesis using M-MLV Reverse Transcriptase Kit (Invitrogen) as per the manufacturer's protocol. 1 $\mu \mathrm{l}$ of $50 \mu \mathrm{M}$ oligod(T) primer (Invitrogen) was used for $20 \mu \mathrm{l}$ cDNA reaction. cDNA (1:20 dilution) was used for RT-qPCR analysis and primer sequences used were as described previously by Datkhile et al. (2011) Hsp70 (for-5' -CATGGCTTGATTCCAACACTCTTGC-3' ; rev-5 ' -CAACTTCCTCAACTGTTGGACCTG-3') and tubulin (for-5' - GCGTGAATGTATCTCAGTTCATGTTG; rev-5' -GACGTGCTTTCCAGCACCAGT-3'). $6 \mu \mathrm{l}$ of diluted cDNA, $1 \mu \mathrm{l}$ of $10 \mu \mathrm{M}$ primer mix (including forward and reverse primer), $8 \mu$ l of nuclease free water and $12 \mu \mathrm{l}$ of SYBR Green (Thermo) master mix formed a total reaction mixture of $19 \mu \mathrm{l}$. Thermal cycle parameters used were as follows: $15 \mathrm{~min}$ for initial denaturation and enzyme activation at $95^{\circ} \mathrm{C}, 45$ cycles for $15 \mathrm{~s}$ at 95 ${ }^{\circ} \mathrm{C}, 30 \mathrm{~s}$ at $55^{\circ} \mathrm{C}$ and a final elongation step for $30 \mathrm{~s}$ at $72{ }^{\circ} \mathrm{C}$. Ct values were calculated on the basis of triplicate sets and tubulin gene transcription was used as reference 
for normalization. mRNA levels in the samples were expressed as fold induction relative to the control value (set to 1 ).

\section{Statistics}

All experiments were carried out in three replications. Mean \pm SD values obtained were analysed by Student's t-test (SPSS software, version 12.0; Chicago, Illinois).

\section{Results and Discussion}

\section{Desiccation Tolerance}

Field populations of $C$. ramosus larvae have often been observed to suffer from dehydration during summers (Fig. 1a). The representative field image shows the larval housing tubes of $C$. ramosus inhabiting a rock pool which is in the process of drying up. However, this larval population exhibited the ability to revive after spells of desiccation conditions. This prompted us to explore the capability of desiccation tolerance in the larvae. Therefore, in order to understand the physiological underpinnings of desiccation tolerance in C. ramosus, we subjected the larvae to acute desiccation stress under laboratory conditions. At the end of desiccation, body water content in the larvae reduced drastically (Fig. 1b). When rehydrated, larvae imbibed water and subsequently showed signs of revival with progressive recovery. Next, we identified thiol damage as one of the effects of the desiccation- triggered body water loss (Fig. 1c). Spectrofluorimetric analysis using 5-IAF dye indicated substantial thiol damage in the desiccating larvae. Intensity of thiol damage decreased with progressive recovery in the rehydrating larvae which was indicative of damage restoration. In proteins, thiol groups (also known as mercaptans or sulfhydryl) are found in cysteine residues. In response to changes in internal conditions, they are known to undergo modifications (such as disulphide formation between two thiol groups) (Conte and Caroll 2013). Desiccation-responsive modifications in thiol have been shown in plants (Kranner and Grill 1997). On similar lines, while exploring for changes in thiol in response to desiccation exposure, we have used 5-IAF, the thiol reactive dye which principally detects changes in protein structure. In biological systems, thiol plays a key role in regulating the oxidation-reduction state of proteins in cells and organisms (Winterbourn and Metodiewa 1999). Our results indicated higher signal intensity of the dye in the desiccated samples which subsequently declined towards the later hours of recovery. These data suggested the probable occurrence of thiol-related changes in the larvae due to desiccation. It will therefore be interesting to carry out future studies to look into the status of oxidative stress in the larvae, particularly on the involvement of thiol which in turn could provide insights into the structural response of proteins to desiccation stress. 
larvae, as a consequence of which metamorphosis into the pupal and adult stages was further delayed (Fig. 2a). We did not find any apparent phenotypic abnormalities in the pupae and adults post recovery. Since the third instar larvae were used in our study, we could track their life history through the fourth instar stage followed by the pupal and adult stages. Upon rehydration, we observed $22 \%$ mortality of the dehydrated larvae while the rest could revive and molt into the fourth instar stage. Of these, around $23 \%$ of the larvae died before pupation. Thus, of the desiccated larvae, an overall mortality of $45 \%$ was seen at the larval stage while 55\% individuals could pupate. Among the pupated individuals, around 10\% died at the pupal stage while the remaining proportion of the pupae metamorphosed into adults. When compared to laboratory reared larvae of C. ramosus, natural population was found to show substantially higher desiccation tolerance threshold (Thorat and Nath, personal observations). In the case of dehydrated larvae obtained from the field, we observed an overall mortality rate of $15 \%$ whereas the rest of the individuals could undergo metamorphosis (unpublished data).

C. ramosus larvae exhibited the ability of revival and resumption of metabolism upon return of favourable hydrating conditions. However, this ability was seen to be associated with a trade-off. The trade-off involved in this case was the delay in metamorphosis of the larvae into pupae and subsequently into adults. Interestingly, this developmental heterochrony appeared to be linked to the duration of desiccation exposure. As seen in Fig. 2a, with increasing exposure time, temporal changes in the developmental profile were more pronounced. Temporal perturbations in development can be fatal for organisms in their natural habitats. In natural settings, if a particular developmental stage sustains for a shorter or longer period than normal, it is quite likely that the next developmental stage might miss the optimal seasonal conditions appropriate for its survival, feeding and reproduction. In addition, large amount of energetic reserves are expected to be invested during recovery from desiccation stress. This also might affect the overall fitness of the revived individuals, in terms of shortfalls of energy allocations for other life activities including reproduction.

Stressful environments determine the temporal patterns of development in organisms and uncoordinated metamorphosis could pose detrimental risks to organisms. In this regard, ecdysone, a steroid hormone from the insect prothoracic gland is converted into 20-hydroxyecdysone in target tissues which dictates the transition of larval-pupal state. We therefore found it worthwhile to look into the ecdysone hormone titres of the larvae which would confirm the involvement of hormonal signals in response to desiccation stress. We indeed found that the larvae desiccated for $60 \mathrm{~min}$ showed variations in the developmental calendar by lengthening of the larval period. Ecdysone analysis by LC-MS confirmed that ecdysone was indeed present in lower amounts in the treated larvae as compared to the untreated controls which showed normal metamorphosis and development (Figs. 2b-d). With favourable conditions, larvae showed increasing levels of ecdysone suggesting their entry into the active phase wherein normal development and life activities could be continued. The low levels of ecdysone in the dry larvae indicate hypo-activity in the absence of metabolic water. Thus desiccation stress is capable of leading to developmental heterochrony in the larvae of C. ramosus. Insect molting is initiated with the release of prothoracicotropic hormone (PTTH) that stimulates the prothoracic glands for the production of ecdysteroids (Hahn and Denlinger 2011). It has also been established that diapause in insects is partly governed by the inhibition of PTTH release, thereby ensuring a block in molting and subsequent pupation. Apart from the hormonal control, photoperiod regulation of insect diapause has received appreciable attention. In this context, the expression of circadian clock genes, namely, period and Clock have been shown to be strongly up-regulated in diapausing adults of certain arthropods such as Pyrrhocoris apterus (Syrová et al. 2003; Nylin 2013). In the past, similar observations from our laboratory have shown the effects of dietary iron supply on the developmental profile in C. ramosus wherein larvae with lower dietary iron content showed erratic and sporadic patterns of eclosion resulting in lengthening of the pre-metamorphic stages (Nath and Babrekar 2009). Further studies revealing the mechanism of the crucial players involved in such desiccation-induced developmental heterochrony could provide useful insights into the consequences, survival and distribution of C. ramosus during dehydrating bouts in natural habitats.

\section{Hsp70 Analysis}

Results from RTqPCR analysis showed that Hsp70 mRNA expression in the control and desiccated larvae of C. ramosus remained almost uniform (Fig. 3). We observed that Hsp70 levels were elicited during the early hours of rehydration and reached the maximum value with a 4.5 fold induction at $3 \mathrm{~h}$ of rehydration as compared to the control. Consequently, with progressive recovery, levels of Hsp70 underwent further decline and matched the control value by $7 \mathrm{~h}$ of rehydration. Elevated expression of Hsp70 in the rehydrating larvae indicated the involvement of Hsp70 in alleviating the effects of desiccation in of $C$. ramosus larvae. A previous study has also shown that larvae of C. ramosus showed higher levels of Hsp70 post irradiation recovery (Datkhile et al. 2011). In insects, the significance of Hsp genes has been well established during biotic as well as abiotic stress conditions, namely, parasitic infections, pathogen attack, temperature, UV radiation, dehydration and chemical and metals (see Zao and Jones 2012 for details). In the case of dehydration, up-regulation of Hsp genes has been shown to be highly responsive in other chironomid midges including Polypedilum vanderplanki and Belgica antarctica (Lopez-Martinez et 


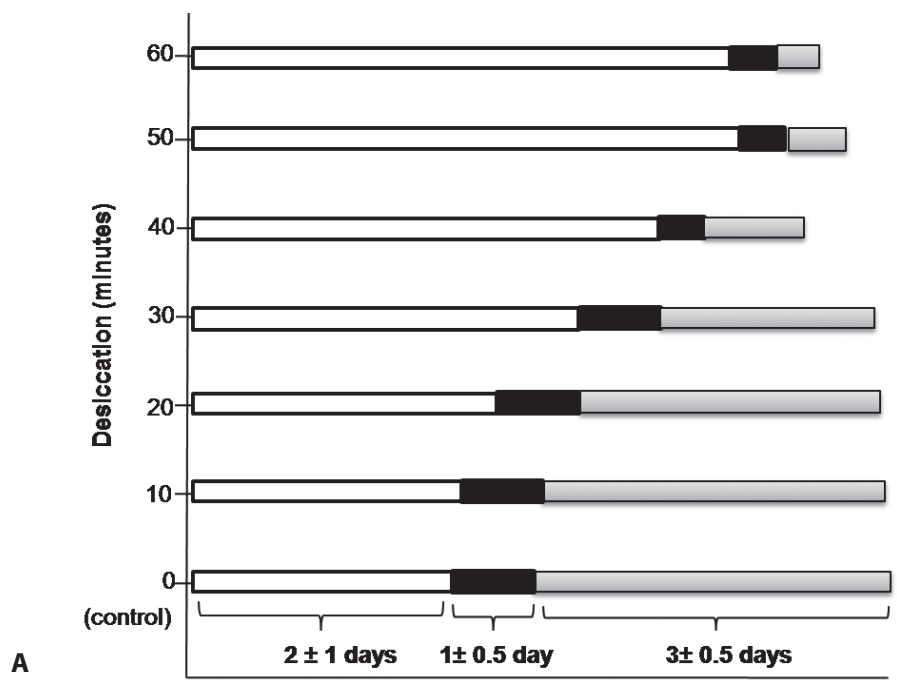

(b) Control

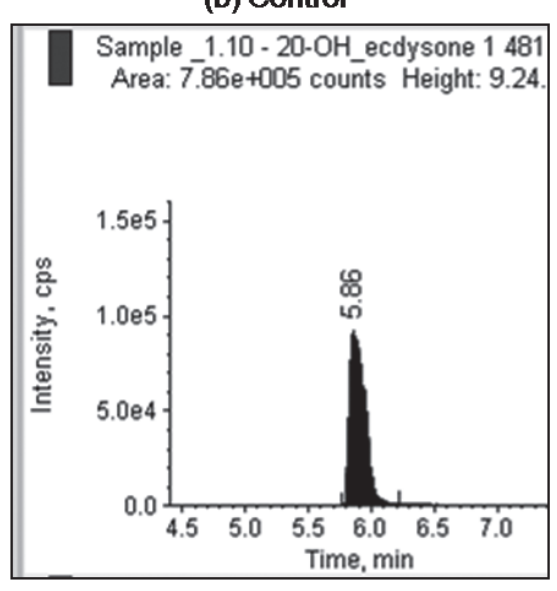

B (c) Desiccated

Sample_1.2... 20-OH_ecdysone 14... Area: $\overline{1} .67 \mathrm{e}+005$ counts Height: 2.8 ...

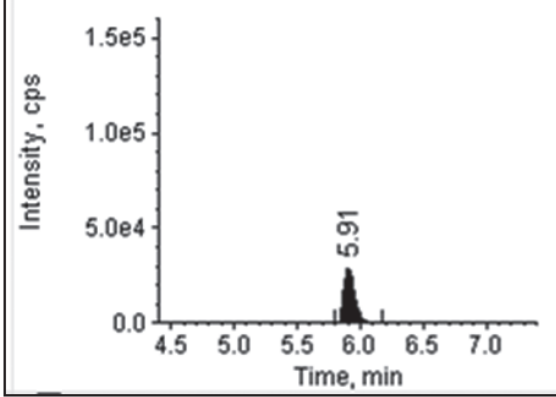

C (d) Rehydrated

Sample_White ext - 20-OH_ecdysone Area: $\overline{7} .80 \mathrm{e}+005$ counts Height: 1.07

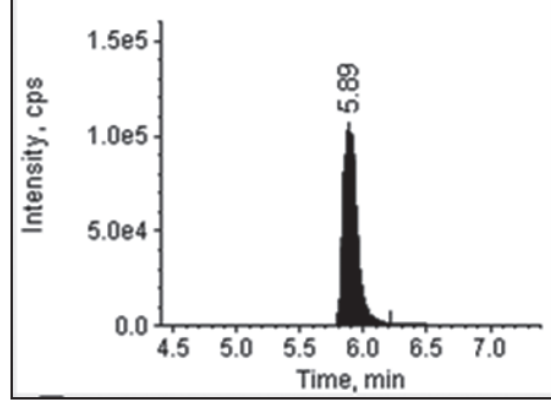

D

Fig. 2 Developmental profile of the larvae post desiccation a) schematic depicting the life span and development upon rehydration - larvae (white), pupae (black), adults (grey); b-d) representative chromatograms of ecdysone hormone titres in the larvae. b) control; c) desiccated; d) rehydrated.

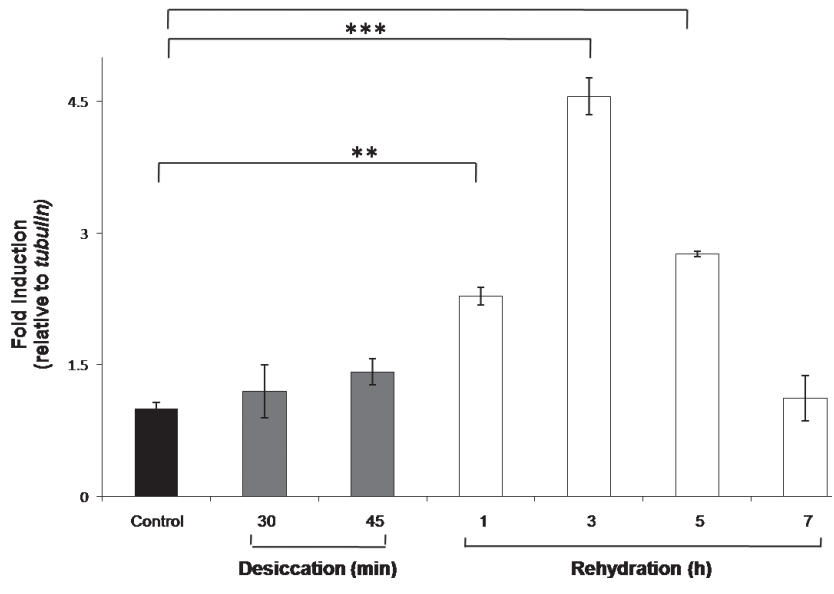

Fig. 3 RT-qPCR analysis for detection of Hsp70 expression. Values indicate fold induction relative to tubulin. Hsp70 expression was elicited during the first few hours of rehydration with a subsequent decline with progressive recovery (Student's t-test: $\mathrm{df}=4, \mathrm{p}<0.005\left(t_{1 \mathrm{~h} \text { rehydration }}=\right.$ $\left.18.16), \mathrm{p}<0.0001\left(t 3_{\mathrm{h} \text { rehydration }}=40.02, t_{5 \mathrm{~h} \text { rehydration }}=24.32\right)\right)$.

al. 2009; Gusev et al. 2011). A prominent rise in Hsp70 levels during the recovery phase has also been reported in desiccation tolerant tardigrades (Jönsson and Schill 2007). The authors thus conclude that the role of Hsp70 is essential for post-desiccation activities of cell revival rather than protection of damage during desiccation. Among the mosquito species Aedes aegypti, Anopheles gambiae and Culex pipiens, a differential response of Hsp70 expression was observed during dehydration (Benoit et al. 2009). Thus it is more likely that the desiccation-responsive expression of Hsp70 occurs in a taxon-specific manner.

\section{Conclusions}

We show that larvae of C. ramosus are capable of desiccation tolerance upon exposure to low RH conditions. Larvae exhibit a delayed pattern of developmental calendar by lengthening the larval stage post rehydration. Validation of these data came from quantitative analysis of ecdysone titres wherein the desiccated larvae showed lower levels of this hormone. These data suggested the organism's plasticity in developmental homeostasis when encountering dehydration stress. We also show the involvement of Hsp70 gene which is one of the most evolutionarily conserved stress protectants. In our study 
Hsp70 response was triggered during the recovery phase in the larvae. Thus it seems that C. ramosus larvae possess the conserved protective mechanism involving Hsp70 to overcome the damages inflicted due to dehydration. Complex physiological and biochemical adjustments involving different molecular players in desiccation tolerant organisms dictate the extent of their survival and recovery from water loss. Therefore investigations directed for the identification of other stress-responsive genes would be essential to get a holistic view of the ongoing recovery mechanisms in the larvae of $C$. ramosus.

\section{Acknowledgements}

We are extremely grateful to Dr Takashi Okuda for helpful advice and critical suggestions. Thanks are also due to Dr Kaushik Banerjee and Dr Dashrath Oulkar for their valuable help and expertise in chromatographic analysis and to Dr Suvro Chatterjee and Krishnapriya for spectrofluorometry. This work is supported by the partial funding from UGC-CAS (Phase-II) and DST-PURSE to the Department of Zoology. BBN is grateful to the financial assistance from the UoP-BCUD grant (OSD/BCUD/360/18). LJT acknowledges the financial support received from the Council of Scientific and Industrial Research - Senior Research Fellowship (CSIR-SRF), New Delhi, India.

\section{REFERENCES}

Benoit JB, Lopez-Martinez G, Phillips ZP, Patrick KR, Denlinger DL (2010) Heat shock proteins contribute to mosquito dehydration tolerance. J Insect Physiol 56: 151-156.

Cao C, Wang Z, Niu C, Desneux N, Gao X (2013) Transcriptome Profiling of Chironomus kiiensis under Phenol Stress Using Solexa Sequencing Technology. PLoS ONE 8: e58914.

Choi J, Ha MH (2009) Effect of cadmium exposure on the globin protein expression in 4th instar larvae of Chironomus riparius Mg. (Diptera: Chironomidae): an ecotoxicoproteomics approach. Proteomics 9: 31-9.

Chown SL, Sørense JG, Terblanche JS (2011) Water loss in insects: An environmental change perspective. J Insect Physiol 57: 1070-1084.

Conte ML, Carroll KS (2013) The Chemistry of Thiol Oxidation and Detection. In: Jakob U, Reichmann D (eds) Oxidative Stress and Redox Regulation, Springer, pp. 1-42.

Datkhile K, Mukhopadhyaya R, Dongre TK, Nath BB (2011) Hsp70 expression in Chironomus ramosus exposed to gamma radiation. Int J Rad Biol 87: 213-221.

Ferrington LC Jr (2008) Global diversity of non-biting midges (Chironomidae: Diptera in fresh water. Hydrobiologia 595: 447-455.

Gusev O, Cornette R, Kikawada T, Okuda T (2011) Expression of heat shock protein-coding genes associated with anhydrobiosis in an African chironomid Polypedilum vanderplanki. Cell Stress Chaperones 16: 81-90.

Hahn DA, Denlinger DL (2011) Energetics of Insect Diapause. Ann Rev Entomol 56: 103-121.

Jönsson KI, Schill RO (2007) Induction of Hsp70 by desiccation, ionising radiation and heat-shock in the eutardigrade Richtersius coronifer. Comp Biochem Physiol Part B 146:।
$456-460$.

Koštál V (2006) Eco-physiological phases of insect diapauses. J Insect Physiol 52: 113-127.

Kranner I, Grill D (1997) Desiccation and the Subsequent Recovery of Cryptogamics that are Resistant to Drought. Phyton 37: 139-150.

Lopez-Martinez G, Benoit JB, Rinehart JP, Elnitsky MA, Lee RE, Denlinger DL (2009) Dehydration, rehydration, and overhydration alter patterns of gene expression in the Antarctic midge, Belgica antarctica. J Comp Physiol B 179: 481-491.

Menge BA, Olson AM, Dahlhoff EP (2002) Environmental Stress, Bottom-up Effects, and Community Dynamics: Integrating Molecular-Physiological and Ecological Approaches. Integ and Comp Biol 42: 892-908.

Morais GS, Pesenti EC, Cestari MM, Navarro-Silva MA (2014) Genotoxic effect of Phenanthrene on Chironomus sancticaroli (Diptera: Chironomidae). Zoologica 31: 323-328.

Nath BB, Godbole NN (1998) Technique for mass rearing of Indian Chironomus species. Studia Dipterologica 5: 187-193.

Nath BB, Babrekar AA (2009) Implications of hypoxic stress in Chironomus ramosus larvae with reference to iron. In: Wang X, Liu W (eds) Contemporary Chironomid Studies - Proceedings of the 17th International Symposium on Chironomidae. Nankai University Press, pp. 334-346.

Nylin S (2013) Induction of diapuase and seasonal morphs in butterflies and other insects: knowns, unknowns and the challenge of integration. Physiol Entomol 38: 96-104.

Oostra V, de Jong MA, Invergo BM et al. (2011) Translating environmental gradients into discontinuous reaction norms via hormone signalling in a polyphenic butterfly. Proc Biol Sci 278: 789-797.

Raushenbach IY, Gruntenko NE, Bownes M et al. (2004) The role of juvenile hormone in the control of reproductive function in Drosophila virilis under nutritional stress. J Insect Physiol 50: 323-330.

Rosenberg DM (1992) Freshwater biomonitoring and Chironomidae. Netherland J Aq Ecol 26: 101-122.

Singtripop T, Oda Y, Wanichacheewa S, Sakurai S (2002) Sensitivities to juvenile hormone and ecdysteroid in the diapauses larvae of Omphisa fuscidentalis based on the hemolymph trehalose dynamics index. J Insect Physiol 48: 817-824.

Smith KK (2003) Time's arrow: heterochrony and the evolution of development. Int J Dev Biol 47: 613-621.

Syrová Z, Doležel D, Šauman I, Hodková M (2003) Photoperiod regulation of diapuse in linden bugs: are period and Clock genes involved? Cell Mol Life Sci 60: 2510-2515.

Thorat JT, Gaikwad S, Nath BB (2012) Trehalose as an indicator of desiccation stress in Drosophila melanogaster larvae: A potential marker of anhydrobiosis. Biochem Biophys Res Comm 419: 638-642.

Thorat L, Nath BB (2010) Trends in Chironomid Research. Chironomus 23: 34-35.

Tunnacliffe A, Lapinski J (2003) Resurrecting Van Leeuwenhoek's rotifers: a reappraisal of the role of disaccharides in anhydrobiosis. Phil Trans R Soc Lond B 358: 1755-1771.

Vandersmissen HP, Van Hiel MB, Van Loy T, Vleugels R, Vanden Broeck J (2014) Silencing D. melanogaster lgr1 impairs transition from larval to pupal stage. Gen Comp Endocrinol doi: http://dx.doi.org/10.1016/j.ygcen.2014.08.006.

Winterbourn CC, Metodiewa D (1999) Reactivity of biologically important thiol compounds with superoxide and hydrogen peroxide. Free Rad Biol Med 27: 322-328.

Zhao L, Jones WA (2012) Expression of heat shock protein genes in insect stress responses. Invert Sur J 9: 93-101. 